\title{
NOTES ON ENDPOINT ESTIMATES FOR MULTILINEAR FRACTIONAL INTEGRAL OPERATORS
}

\author{
YASUO KOMORI-FURUYA
}

(Communicated by Alexander Iosevich)

\begin{abstract}
We prove the strong type estimates for the multilinear fractional integral operators of Kenig and Stein type at critical indices. Our results are optimal.
\end{abstract}

\section{INTRODUCTION}

Since Lacey and Thiele [5] proved the $L^{p}$ boundedness of the bilinear Hilbert transform, and Grafakos and Torres [2] proved the $L^{p}$ boundedness of multilinear singular integrals, considerable attention has been paid to the study of multilinear operators. Kenig and Stein [4] proved the boundedness of multilinear fractional integral operators on $L^{p}$ spaces and weak type estimates on $L^{1}$. Tang [6] showed the BMO estimates at critical indices for multilinear fractional integral operators. In this paper we consider strong type estimates on $L^{1}$ and $L^{\infty}$ estimates at critical indices. We also show that our results are optimal by giving counterexamples.

Ordinary fractional integral operators are defined by

$$
I_{\alpha} f(x)=\int_{\mathbb{R}^{n}} \frac{f(y)}{|x-y|^{n-\alpha}} d y \quad \text { where } \quad 0<\alpha<n .
$$

We know the following (see, for example, [1]):

$$
\begin{aligned}
& I_{\alpha}: L^{p} \rightarrow L^{q} \quad \text { when } \quad \frac{1}{q}=\frac{1}{p}-\frac{\alpha}{n}>0 \text { and } p>1, \\
& I_{\alpha}: L^{1} \rightarrow L^{q, \infty} \text { when } \quad \frac{1}{q}=1-\frac{\alpha}{n}, \\
& I_{\alpha}: L^{n / \alpha} \nrightarrow L^{\infty} .
\end{aligned}
$$

We define the multilinear fractional integral operators.

\section{Definition 1.}

$$
I_{m, \alpha}\left(f_{1}, \ldots, f_{m}\right)(x):=\int_{\left(\mathbb{R}^{n}\right)^{m}} \frac{f_{1}\left(y_{1}\right) \cdots f_{m}\left(y_{m}\right)}{\left(\left|x-y_{1}\right|+\cdots+\left|x-y_{m}\right|\right)^{m n-\alpha}} d y_{1} \cdots d y_{m},
$$

where $m \geq 2$ and $0<\alpha<m n$.

Remark. Kenig and Stein [4] define $I_{m, \alpha}$ by

$$
\int f_{1}\left(y_{1}\right) \cdots f_{m}\left(y_{m}\right) /\left(\left|x-y_{1}\right|^{2}+\cdots+\left|x-y_{m}\right|^{2}\right)^{-(m n-\alpha) / 2} d y_{1} \cdots d y_{m},
$$

but we use our definition for simplicity of notation.

Received by the editors August 11, 2013.

2010 Mathematics Subject Classification. Primary 42B20.

Key words and phrases. Fractional integral, multilinear fractional integral, endpoint estimate. 
We know the following two results.

Theorem (Kenig and Stein [4]). Let

$$
\frac{1}{q}=\sum_{i=1}^{m} \frac{1}{p_{i}}-\frac{\alpha}{n}>0 .
$$

If each $p_{i}>1$, then $I_{m, \alpha}$ is bounded from $L^{p_{1}}\left(\mathbb{R}^{n}\right) \times \cdots \times L^{p_{m}}\left(\mathbb{R}^{n}\right)$ to $L^{q}\left(\mathbb{R}^{n}\right)$. If $p_{i}=1$ for some $i$, then $I_{m, \alpha}$ is bounded from $L^{p_{1}}\left(\mathbb{R}^{n}\right) \times \cdots \times L^{p_{m}}\left(\mathbb{R}^{n}\right)$ to $L^{q, \infty}\left(\mathbb{R}^{n}\right)$.

Remark. A simple example shows that $I_{m, \alpha}$ is not bounded from $L^{1} \times \cdots \times L^{1}$ to $L^{q}$ where $1 / q=m-\alpha / n$ (see the counterexample (19)).

Theorem (Tang [6]). If $\sum_{i=1}^{m} 1 / p_{i}=\alpha / n$, then $I_{m, \alpha}$ is bounded from $L^{p_{1}}\left(\mathbb{R}^{n}\right) \times$ $\cdots \times L^{p_{m}}\left(\mathbb{R}^{n}\right)$ to $B M O\left(\mathbb{R}^{n}\right)$.

Note that $L^{\infty} \subsetneq B M O$.

In this paper we shall prove that $I_{m, \alpha}$ is bounded from $\prod_{i=1}^{m} L^{p_{i}}$ to $L^{q}$ even if some $p_{i}$ are equal to one under additional conditions on $\alpha$. Furthermore we shall consider $L^{\infty}$ estimates for $I_{m, \alpha}$.

\section{THEOREMS}

We state our results. In the following we always assume that $1 \leq p_{1} \leq p_{2} \leq$ $\cdots \leq p_{m} \leq \infty$.

Theorem 1. Let $p_{1}=\cdots=p_{k}=1$ and $1<p_{k+1}, \ldots, p_{m}<\infty$ for some $1 \leq k \leq$ $m-1$, and

$$
\frac{1}{q}=k+\sum_{i=k+1}^{m} \frac{1}{p_{i}}-\frac{\alpha}{n}>0 .
$$

Assume that

$$
k n \leq \alpha<m n .
$$

Then

$$
\left\|I_{m, \alpha}\left(f_{1}, \ldots, f_{m}\right)\right\|_{q} \leq C \prod_{i=1}^{k}\left\|f_{i}\right\|_{1} \prod_{i=k+1}^{m}\left\|f_{i}\right\|_{p_{i}} .
$$

Throughout this paper, $C$ is a positive constant which is independent of essential parameters and not necessarily the same at each occurrence.

Remark. As we shall see in the proof, the main interest of the theorem is the case when $\alpha=k n$.

The following corollary is easily obtained from Theorem 1.

Corollary 1. Let $p_{1}=\cdots=p_{k}=1,1<p_{k+1}, \ldots, p_{m-l}<\infty$ and $p_{m-l+1}=\cdots=$ $p_{m}=\infty$ for some $0 \leq k<m-l$, and

$$
\frac{1}{q}=k+\sum_{i=k+1}^{m-l} \frac{1}{p_{i}}-\frac{\alpha}{n}>0 .
$$

Assume that

$$
k n \leq \alpha<(m-l) n .
$$


Then

$$
\left\|I_{m, \alpha}\left(f_{1}, \ldots, f_{m}\right)\right\|_{q} \leq C \prod_{i=1}^{k}\left\|f_{i}\right\|_{1} \prod_{i=k+1}^{m-l}\left\|f_{i}\right\|_{p_{i}} \prod_{i=m-l+1}^{m}\left\|f_{i}\right\|_{\infty} .
$$

If $k=0$, then we assume that $1<p_{i}$ for all $i$. If $l=0$, then we assume that $p_{i}<\infty$ for all $i$.

Remark. $I_{m, \alpha}$ is not bounded from $L^{1} \times \cdots \times L^{1} \times L^{\infty} \times \cdots \times L^{\infty}$ to $L^{q}$ (see the counterexamples (20) and (21)).

When $q=\infty$ we obtain the following result.

Theorem 2. Let

Assume that

$$
\sum_{i=1}^{m} \frac{1}{p_{i}}=\frac{\alpha}{n} \quad \text { and } \quad 1<p_{i}<\infty
$$

$$
n \leq \alpha<m n
$$

Then

$$
\left\|I_{m, \alpha}\left(f_{1}, \ldots, f_{m}\right)\right\|_{\infty} \leq C \prod_{i=1}^{m}\left\|f_{i}\right\|_{p_{i}} .
$$

The following corollary is easily obtained from Theorem 2 .

Corollary 2. Let $p_{1}=\cdots=p_{k}=1,1<p_{k+1}, \ldots, p_{m-l}<\infty$ and $p_{m-l+1}=\cdots=$ $p_{m}=\infty$ for some $0 \leq k<m-l-1$, and

$$
k+\sum_{i=k+1}^{m-l} \frac{1}{p_{i}}=\frac{\alpha}{n} .
$$

Assume that

$$
(k+1) n \leq \alpha<(m-l) n .
$$

Then

$$
\left\|I_{m, \alpha}\left(f_{1}, \ldots, f_{m}\right)\right\|_{\infty} \leq C \prod_{i=1}^{k}\left\|f_{i}\right\|_{1} \prod_{i=k+1}^{m-l}\left\|f_{i}\right\|_{p_{i}} \prod_{i=m-l+1}^{m}\left\|f_{i}\right\|_{\infty} .
$$

If $k=0$, then we assume that $1<p_{i}$ for all $i$. If $l=0$, then we assume that $p_{i}<\infty$ for all $i$.

Remark. $I_{m, \alpha}$ is not bounded from $L^{1} \times \cdots \times L^{1} \times L^{p_{i}} \times L^{\infty} \times \cdots \times L^{\infty}$ to $L^{\infty}$ (see the counterexample (27)).

We shall show that the conditions (10) (4) are optimal by giving counterexamples in Section 4.

Proof of Corollary 1. We may assume that $f_{i} \geq 0$ for all $i$. Since

$$
\begin{aligned}
& \int_{\left(\mathbb{R}^{n}\right)^{l}} \frac{1}{\left(\left|x-y_{m-l+1}\right|+\left|x-y_{m-l+2}\right|+\cdots+\left|x-y_{m}\right|+A\right)^{m n-\alpha}} d y_{m-l+1} \cdots d y_{m} \\
& \leq \frac{C}{A^{m n-\alpha-l n}} \quad \text { where } \quad A>0
\end{aligned}
$$


we have

$$
\begin{aligned}
& I_{m, \alpha}\left(f_{1}, \ldots, f_{m}\right)(x) \\
& \leq \prod_{i=m-l+1}^{m}\left\|f_{i}\right\|_{\infty} \int_{\left(\mathbb{R}^{n}\right)^{m-l}} \frac{f_{1}\left(y_{1}\right) \cdots f_{m-l}\left(y_{m-l}\right)}{\left(\left|x-y_{1}\right|+\cdots+\left|x-y_{m-l}\right|\right)^{m n-\alpha-l n}} d y_{1} \cdots d y_{m-l} \\
& \leq \prod_{i=m-l+1}^{m}\left\|f_{i}\right\|_{\infty} I_{m-l, \alpha}\left(f_{1}, \ldots, f_{m-l}\right)(x) .
\end{aligned}
$$

Note that $k n \leq \alpha<(m-l) n$ and $1 / q=k+\sum_{i=k+1}^{m-l} 1 / p_{i}-\alpha / n$. By Theorem 1 or Theorem (Kenig and Stein) if $k=0$, we have

$$
\left\|I_{m-l, \alpha}\left(f_{1}, \ldots, f_{m-l}\right)\right\|_{q} \leq C \prod_{i=1}^{k}\left\|f_{i}\right\|_{1} \prod_{i=k+1}^{m-l}\left\|f_{i}\right\|_{p_{i}} .
$$

Proof of Corollary 2. We may assume that $f_{i} \geq 0$ for all $i$. Then

$$
\begin{aligned}
& I_{m, \alpha}\left(f_{1}, \ldots, f_{m}\right)(x) \\
& \leq C \prod_{i=1}^{k}\left\|f_{i}\right\|_{1} \prod_{i=m-l+1}^{m}\left\|f_{i}\right\|_{\infty} \\
& \quad \times \int_{\left(\mathbb{R}^{n}\right)^{m-k-l}} \frac{f_{k+1}\left(y_{k+1}\right) \cdots f_{m-l}\left(y_{m-l}\right)}{\left(\left|x-y_{k+1}\right|+\cdots+\left|x-y_{m-l}\right|\right)^{m n-\alpha-l n}} d y_{k+1} \cdots d y_{m-l} \\
& \leq C \prod_{i=1}^{k}\left\|f_{i}\right\|_{1} \prod_{i=m-l+1}^{m}\left\|f_{i}\right\|_{\infty} I_{m-k-l, \alpha-k n}\left(f_{k+1}, \ldots, f_{m-l}\right)(x) .
\end{aligned}
$$

Since $n \leq \alpha-k n<(m-k-l) n$ and $\sum_{i=k+1}^{m-l} 1 / p_{i}=(\alpha-k n) / n$, we have by Theorem 2

$$
\left\|I_{m-k-l, \alpha-k n}\left(f_{k+1}, \ldots, f_{m-l}\right)\right\|_{\infty} \leq C \prod_{i=k+1}^{m-l}\left\|f_{i}\right\|_{p_{i}} .
$$

\section{Proofs of theorems}

This section is organized as follows. First we prove Theorem 1 where $k n<\alpha<$ $m n$. This is easy. For Theorem 2 and Theorem 1 where $k n=\alpha$, we prove them only for $m=2$ and $m=3$, because when $m \geq 4$ we can prove them by induction on $m$. Finally we show outlines of proofs when $m \geq 4$.

In the following we assume that $f_{i} \geq 0$ for all $i$.

Proof of Theorem 1 where $\alpha>k n$. When $k<m-1$, we have

$$
\begin{aligned}
& I_{m, \alpha}\left(f_{1}, \ldots, f_{m}\right)(x) \\
& \leq \prod_{i=1}^{k}\left\|f_{i}\right\|_{1} \int_{\left(\mathbb{R}^{n}\right)^{m-k}} \frac{f_{k+1}\left(y_{k+1}\right) \cdots f_{m}\left(y_{m}\right)}{\left(\left|x-y_{k+1}\right|+\cdots+\left|x-y_{m}\right|\right)^{m n-\alpha}} d y_{k+1} \cdots d y_{m} \\
& \leq \prod_{i=1}^{k}\left\|f_{i}\right\|_{1} I_{m-k, \alpha-k n}\left(f_{k+1}, \ldots f_{m}\right)(x) .
\end{aligned}
$$


Since $0<\alpha-k n<(m-k) n$ and $1 / q=\sum_{i=k+1}^{m} 1 / p_{i}-(\alpha-k n) / n$, we have by Theorem (Kenig and Stein)

$$
\left\|I_{m-k, \alpha-k n}\left(f_{k+1}, \ldots, f_{m}\right)(x)\right\|_{q} \leq C \prod_{i=k+1}^{m}\left\|f_{i}\right\|_{p_{i}} .
$$

When $k=m-1$, we have $I_{m, \alpha}\left(f_{1}, \ldots, f_{m}\right)(x) \leq \prod_{i=1}^{m-1}\left\|f_{i}\right\|_{1} I_{\alpha-k n}\left(f_{m}\right)(x)$, where $I_{\alpha-k n}$ is the ordinary fractional integral operator. Since $0<\alpha-k n<n$ and $1 / q=1 / p_{m}-(\alpha-k n) / n$, we have by the boundedness of $I_{\alpha-k n}$,

$$
\left\|I_{\alpha-k n}\left(f_{m}\right)\right\|_{q} \leq C\left\|f_{m}\right\|_{p_{m}} .
$$

Now we consider the cases when $m=2$ and $m=3$. First we prove Theorem 2 and then we prove Theorem 1 where $k n=\alpha$ by using Theorem 2 .

Theorem $2(\boldsymbol{m}=\mathbf{2})$. Let $1<p_{1}, p_{2}<\infty$ and $1 / p_{1}+1 / p_{2}=\alpha / n$ where $n \leq \alpha<$ $2 n$. Then the bilinear fractional integral operator $I_{2, \alpha}$ is bounded from $L^{p_{1}}\left(\mathbb{R}^{n}\right) \times$ $L^{p_{2}}\left(\mathbb{R}^{n}\right)$ to $L^{\infty}\left(\mathbb{R}^{n}\right)$.

Proof. This is essentially proved in [3. Theorem 340, p. 253] when $n=1$. We give a proof for the sake of completeness. Let

$$
J_{2, \alpha}\left(f_{1}, f_{2}\right):=\int_{\left(\mathbb{R}^{n}\right)^{2}} \frac{f_{1}\left(y_{1}\right) f_{2}\left(y_{2}\right)}{\left(\left|y_{1}\right|+\left|y_{2}\right|\right)^{2 n-\alpha}} d y_{1} d y_{2} .
$$

It suffices to show that

$$
J_{2, \alpha}\left(f_{1}, f_{2}\right) \leq C\left\|f_{1}\right\|_{p_{1}}\left\|f_{2}\right\|_{p_{2}} .
$$

We write

$$
J_{2, \alpha}\left(f_{1}, f_{2}\right)=\int_{\left|y_{2}\right| \leq\left|y_{1}\right|}+\int_{\left|y_{2}\right| \geq\left|y_{1}\right|}=: J^{1}+J^{2} .
$$

We estimate $J^{1} . J^{2}$ can be estimated similarly. Since

$$
(-2 n+\alpha) p_{1}^{\prime}+n p_{2}+n\left(p_{1}^{\prime}-p_{2}\right) / p_{2}^{\prime}=0
$$

and $p_{1}^{\prime} \geq p_{2}$, we have

$$
\begin{aligned}
J^{1} & \leq \int_{\mathbb{R}^{n}} \frac{f_{1}\left(y_{1}\right)}{\left|y_{1}\right|^{2 n-\alpha}}\left(\int_{\left|y_{2}\right| \leq\left|y_{1}\right|} f_{2}\left(y_{2}\right) d y_{2}\right) d y_{1} \\
& \leq\left\|f_{1}\right\|_{p_{1}}\left[\int_{\mathbb{R}^{n}} \frac{1}{\left|y_{1}\right|^{(2 n-\alpha) p_{1}^{\prime}}}\left(\int_{\left|y_{2}\right| \leq\left|y_{1}\right|} f_{2}\left(y_{2}\right) d y_{2}\right)^{p_{1}^{\prime}} d y_{1}\right]^{1 / p_{1}^{\prime}} \\
& \leq\left\|f_{1}\right\|_{p_{1}}\left[\int_{\mathbb{R}^{n}}\left|y_{1}\right|^{(-2 n+\alpha) p_{1}^{\prime}+n p_{2}}\left(\frac{1}{\left|y_{1}\right|^{n}} \int_{\left|y_{2}\right| \leq\left|y_{1}\right|} f_{2}\left(y_{2}\right) d y_{2}\right)^{p_{2}}\right. \\
& \leq C\left\|f_{1}\right\|_{p_{1}}\left\|f_{2}\right\|_{p_{2}}^{\left(p_{1}^{\prime}-p_{2}\right) / p_{1}^{\prime}}\left[\int_{\mathbb{R}^{n}}\left(\frac{1}{\left|y_{1}\right|^{n}} \int_{\left|y_{2}\right| \leq\left|y_{1}\right|} f_{2}\left(y_{2}\right) d y_{2}\right)^{p_{1}^{\prime}-p_{2}} d y_{2}\right]^{1 / p_{1}^{\prime}} \\
& \left.\leq C\left\|y_{1}\right\|_{p_{1}}\left\|f_{2}\right\|_{p_{2}}^{\left(p_{1}^{\prime}-p_{2}\right) / p_{1}^{\prime}}\left(\int_{\mathbb{R}^{n}} M f_{2}\left(y_{1}\right)^{p_{2}} d y_{1}\right)^{1 / p_{1}^{\prime}} \leq C y_{1}\right]^{1 / p_{1}^{\prime}} \leq f_{1}\left\|_{p_{1}}\right\| f_{2} \|_{p_{2}},
\end{aligned}
$$

where $M$ is the Hardy-Littlewood maximal operator. 
Theorem $2(\boldsymbol{m}=\mathbf{3})$. Let $1<p_{1} \leq p_{2} \leq p_{3}<\infty, 1 / p_{1}+1 / p_{2}+1 / p_{3}=\alpha / n$ and $n \leq \alpha<3 n$. Then the trilinear fractional integral operator $I_{3, \alpha}$ is bounded from $L^{p_{1}}\left(\mathbb{R}^{n}\right) \times L^{p_{2}}\left(\mathbb{R}^{n}\right) \times L^{p_{3}}\left(\mathbb{R}^{n}\right)$ to $L^{\infty}\left(\mathbb{R}^{n}\right)$.

Proof. Let

$$
J_{3, \alpha}\left(f_{1}, f_{2}, f_{3}\right):=\int_{\left(\mathbb{R}^{n}\right)^{3}} \frac{f_{1}\left(y_{1}\right) f_{2}\left(y_{2}\right) f_{3}\left(y_{3}\right)}{\left(\left|y_{1}\right|+\left|y_{2}\right|+\left|y_{3}\right|\right)^{3 n-\alpha}} d y_{1} d y_{2} d y_{3} .
$$

Note that

$$
\left\{\begin{array} { l } 
{ 1 / p _ { 1 } + 1 / p _ { 2 } = \alpha / n - 1 / p _ { 3 } , } \\
{ 1 / p _ { 1 } + 1 / p _ { 2 } \geq 2 / p _ { 3 } , }
\end{array} \text { and } \quad \left\{\begin{array}{l}
1 / p_{2}+1 / p_{3}=\alpha / n-1 / p_{1}, \\
1 / p_{2}+1 / p_{3} \leq 2 / p_{1} .
\end{array}\right.\right.
$$

By a simple calculation we have the following:

$$
\begin{aligned}
& \text { If } \quad \frac{3 n}{2} \leq \alpha<3 n, \quad \text { then } \quad \frac{1}{p_{1}}+\frac{1}{p_{2}} \geq 1 . \\
& \text { If } \quad n \leq \alpha<\frac{3 n}{2}, \quad \text { then } \quad \frac{1}{p_{2}}+\frac{1}{p_{3}}<1 .
\end{aligned}
$$

The case (7): $3 n / 2 \leq \alpha<3 n$.

By Hölder's inequality we have

$$
\begin{aligned}
J_{3, \alpha}\left(f_{1}, f_{2}, f_{3}\right) & \leq C\left\|f_{3}\right\|_{p_{3}} \int \frac{f_{1}\left(y_{1}\right) f_{2}\left(y_{2}\right)}{\left(\left|y_{1}\right|+\left|y_{2}\right|\right)^{3 n-\alpha-n / p_{3}^{\prime}}} d y_{1} d y_{2} \\
& \leq C\left\|f_{3}\right\|_{p_{3}} J_{2, \alpha-n / p_{3}}\left(f_{1}, f_{2}\right) ;
\end{aligned}
$$

see the definition (5). Since $n \leq \alpha-n / p_{3}<2 n$ and $1 / p_{1}+1 / p_{2}=\left(\alpha-n / p_{3}\right) / n$, we can apply Theorem $2(m=2)$ and we obtain the desired result.

The case (8): $n \leq \alpha<3 n / 2$.

We write

$$
J_{3, \alpha}\left(f_{1}, f_{2}, f_{3}\right)=\int_{\left|y_{3}\right| \leq\left|y_{2}\right|} d y_{1} d y_{2} d y_{3}+\int_{\left|y_{3}\right| \geq\left|y_{2}\right|} d y_{1} d y_{2} d y_{3}=: J^{1}+J^{2} .
$$

It suffices to estimate $J^{1} . J^{2}$ can be estimated similarly. We have

$$
\begin{aligned}
J^{1} & \leq \int \frac{f_{1}\left(y_{1}\right) f_{2}\left(y_{2}\right)}{\left(\left|y_{1}\right|+\left|y_{2}\right|\right)^{2 n-\alpha}}\left(\frac{1}{\left|y_{2}\right|^{n}} \int_{\left|y_{3}\right| \leq\left|y_{2}\right|} f_{3}\left(y_{3}\right) d y_{3}\right) d y_{1} d y_{2} \\
& \leq C \int \frac{f_{1}\left(y_{1}\right) f_{2}\left(y_{2}\right) M f_{3}\left(y_{2}\right)}{\left(\left|y_{1}\right|+\left|y_{2}\right|\right)^{2 n-\alpha}} d y_{1} d y_{2}=C J_{2, \alpha}\left(f_{1}, f_{2} \cdot M f_{3}\right) .
\end{aligned}
$$

If $1 / r=1 / p_{2}+1 / p_{3}$, then $r>1$ and $1 / p_{1}+1 / r=\alpha / n$. Therefore we can apply Theorem $2(m=2)$ and we obtain

$$
J_{2, \alpha}\left(f_{1}, f_{2} \cdot M f_{3}\right) \leq C\left\|f_{1}\right\|_{p_{1}}\left\|f_{2} \cdot M f_{3}\right\|_{r} \leq C \prod_{i=1}^{3}\left\|f_{i}\right\|_{p_{i}} .
$$

We prove Theorem 1 for $\alpha=k n$ where $m=2$ and $m=3$.

Theorem $1(\boldsymbol{m}=\mathbf{2}, \boldsymbol{k}=\mathbf{1})$. Let $1<p_{2}<\infty$. Then the bilinear fractional integral operator $I_{2, n}$ is bounded from $L^{1}\left(\mathbb{R}^{n}\right) \times L^{p_{2}}\left(\mathbb{R}^{n}\right)$ to $L^{p_{2}}\left(\mathbb{R}^{n}\right)$. 
Proof. Let $f_{1} \in L^{1}$ and $f_{2} \in L^{p_{2}}$. For any nonnegative function $g \in L^{p_{2}^{\prime}}$,

$$
\int g(x) I_{2, n}\left(f_{1}, f_{2}\right)(x) d x=\int f_{1}\left(y_{1}\right) d y_{1} \int \frac{f_{2}\left(y_{2}\right) g(x)}{\left(\left|x-y_{1}\right|+\left|x-y_{2}\right|\right)^{n}} d y_{2} d x .
$$

Therefore it suffices to show that

$$
\int \frac{f_{2}\left(y_{2}\right) g(x)}{\left(\left|x-y_{1}\right|+\left|x-y_{2}\right|\right)^{n}} d y_{2} d x \leq C\left\|f_{2}\right\|_{p_{2}}\|g\|_{p_{2}^{\prime}} \quad \text { for all } \quad y_{1} \in \mathbb{R}^{n} .
$$

Note that

$$
\int \frac{f_{2}\left(y_{2}\right) g(x)}{\left(\left|x-y_{1}\right|+\left|x-y_{2}\right|\right)^{n}} d y_{2} d x=\int \frac{f_{2}\left(y_{1}+y_{2}\right) g\left(x+y_{1}\right)}{\left(|x|+\left|x-y_{2}\right|\right)^{n}} d y_{2} d x .
$$

Therefore we need to show that

$$
\int \frac{f_{2}\left(y_{2}\right) g(x)}{\left(|x|+\left|x-y_{2}\right|\right)^{n}} d y_{2} d x \leq C\left\|f_{2}\right\|_{p_{2}}\|g\|_{p_{2}^{\prime}} .
$$

We have

$$
\int \frac{f_{2}\left(y_{2}\right) g(x)}{\left(|x|+\left|x-y_{2}\right|\right)^{n}} d y_{2} d x \leq C \int \frac{f_{2}\left(y_{2}\right) g(x)}{\left(|x|+\left|y_{2}\right|\right)^{n}} d y_{2} d x=C J_{2, n}\left(f_{2}, g\right) ;
$$

see (5) for the definition of $J_{2, n}$. Since $1 / p_{2}+1 / p_{2}^{\prime}=n / n$, we can apply Theorem $2(m=2)$ and we obtain

$$
J_{2, n}\left(f_{2}, g\right) \leq C\left\|f_{2}\right\|_{p_{2}}\|g\|_{p_{2}^{\prime}}
$$

Theorem $1(\boldsymbol{m}=\mathbf{3}, \boldsymbol{k}=\mathbf{1})$. Let $1<p_{2}, p_{3}<\infty$ and $1 / q=1 / p_{2}+1 / p_{3}$. Then the trilinear fractional integral operator $I_{3, n}$ is bounded from $L^{1}\left(\mathbb{R}^{n}\right) \times L^{p_{2}}\left(\mathbb{R}^{n}\right) \times$ $L^{p_{3}}\left(\mathbb{R}^{n}\right)$ to $L^{q}\left(\mathbb{R}^{n}\right)$.

Proof. Note that $q$ may be less than one. Therefore we cannot use the same argument as above. We write

$$
\begin{aligned}
I_{3, n}\left(f_{1}, f_{2}, f_{3}\right)(x) & =\int_{\left|y_{2}\right| \leq\left|y_{3}\right|} \frac{f_{1}\left(x-y_{1}\right) f_{2}\left(x-y_{2}\right) f_{3}\left(x-y_{3}\right)}{\left(\left|y_{1}\right|+\left|y_{2}\right|+\left|y_{3}\right|\right)^{2 n}} d y_{1} d y_{2} d y_{3} \\
& +\int_{\left|y_{2}\right| \geq\left|y_{3}\right|} \frac{f_{1}\left(x-y_{1}\right) f_{2}\left(x-y_{2}\right) f_{3}\left(x-y_{3}\right)}{\left(\left|y_{1}\right|+\left|y_{2}\right|+\left|y_{3}\right|\right)^{2 n}} d y_{1} d y_{2} d y_{3} \\
& =: I^{1}(x)+I^{2}(x) .
\end{aligned}
$$

It suffices to estimate $I^{1}$,

$$
\begin{aligned}
I^{1}(x) & \leq \int\left(\frac{1}{\left|y_{3}\right|^{n}} \int_{\left|y_{2}\right| \leq\left|y_{3}\right|} f_{2}\left(x-y_{2}\right) d y_{2}\right) \frac{f_{1}\left(x-y_{1}\right) f_{3}\left(x-y_{3}\right)}{\left(\left|y_{1}\right|+\left|y_{3}\right|\right)^{n}} d y_{1} d y_{3} \\
& \leq C M f_{2}(x) \cdot I_{2, n}\left(f_{1}, f_{3}\right)(x) .
\end{aligned}
$$

We have by Theorem $1(m=2, k=1)$

$$
\left\|I_{2, n}\left(f_{1}, f_{3}\right)\right\|_{p_{3}} \leq C\left\|f_{1}\right\|_{1}\left\|f_{3}\right\|_{p_{3}} .
$$

Therefore we obtain

$$
\left\|I^{1}\right\|_{q} \leq\left\|M f_{2}\right\|_{p_{2}}\left\|I_{2, n}\left(f_{1}, f_{3}\right)\right\|_{p_{3}} \leq C\left\|f_{1}\right\|_{1}\left\|f_{2}\right\|_{p_{2}}\left\|f_{3}\right\|_{p_{3}} .
$$

Theorem $1(\boldsymbol{m}=\mathbf{3}, \boldsymbol{k}=\mathbf{2})$. Let $1<p_{3}<\infty$. Then the trilinear fractional integral operator $I_{3,2 n}$ is bounded from $L^{1}\left(\mathbb{R}^{n}\right) \times L^{1}\left(\mathbb{R}^{n}\right) \times L^{p_{3}}\left(\mathbb{R}^{n}\right)$ to $L^{p_{3}}\left(\mathbb{R}^{n}\right)$. 
Proof. We use the same argument as in the proof of Theorem $1(m=2, k=1)$. We will show that for any nonnegative function $g \in L^{p_{3}^{\prime}}$,

$$
\begin{aligned}
& \int \frac{f_{2}\left(y_{2}\right) f_{3}\left(y_{3}\right) g(x)}{\left(\left|x-y_{1}\right|+\left|x-y_{2}\right|+\left|x-y_{3}\right|\right)^{n}} d y_{2} d y_{3} d x \\
& \leq C\left\|f_{2}\right\|_{1}\left\|f_{3}\right\|_{p_{3}}\|g\|_{p_{3}^{\prime}} \text { for all } y_{1} \in \mathbb{R}^{n} .
\end{aligned}
$$

It suffices to show the following:

$$
\int \frac{f_{2}\left(y_{2}\right) f_{3}\left(y_{3}\right) g(x)}{\left(|x|+\left|x-y_{2}\right|+\left|x-y_{3}\right|\right)^{n}} d y_{2} d y_{3} d x \leq C\left\|f_{2}\right\|_{1}\left\|f_{3}\right\|_{p_{3}}\|g\|_{p_{3}^{\prime}} .
$$

Therefore it is sufficient to show that

$$
\int \frac{f_{3}\left(y_{3}\right) g(x)}{\left(|x|+\left|x-y_{2}\right|+\left|x-y_{3}\right|\right)^{n}} d y_{3} d x \leq C\left\|f_{3}\right\|_{p_{3}}\|g\|_{p_{3}^{\prime}} \quad \text { for all } \quad y_{2} \in \mathbb{R}^{n} .
$$

We have

$$
\begin{aligned}
\int \frac{f_{3}\left(y_{3}\right) g(x)}{\left(|x|+\left|x-y_{2}\right|+\left|x-y_{3}\right|\right)^{n}} d y_{3} d x & \leq C \int \frac{f_{3}\left(y_{3}\right) g(x)}{\left(\left|y_{2}-y_{3}\right|+\left|y_{2}-x\right|\right)^{n}} d y_{3} d x \\
& =C I_{2, n}\left(f_{3}, g\right)\left(y_{2}\right) .
\end{aligned}
$$

Since $1 / p_{3}+1 / p_{3}^{\prime}=n / n$, we can apply Theorem $2(m=2)$ and we obtain the desired result.

When $m \geq 4$, we repeat the same argument as above, therefore we show only outlines of proofs.

An outline of the proof of Theorem 2 for $m \geq 4$. Let

$$
J_{m, \alpha}\left(f_{1}, \ldots, f_{m}\right):=\int_{\left(\mathbb{R}^{n}\right)^{m}} \frac{f_{1}\left(y_{1}\right) \cdots f_{m}\left(y_{m}\right)}{\left(\left|y_{1}\right|+\cdots+\left|y_{m}\right|\right)^{m n-\alpha}} d y_{1} \cdots d y_{m} .
$$

It suffices to show that

$$
J_{m, \alpha}\left(f_{1}, \ldots, f_{m}\right) \leq C \prod_{i=1}^{m}\left\|f_{i}\right\|_{p_{i}} \quad \text { where } \quad n \leq \alpha<m n .
$$

We use induction on $m$. We assume that (12) holds for some $m \geq 3$. Let

$$
\sum_{i=1}^{m+1} \frac{1}{p_{i}}=\frac{\alpha}{n} \quad \text { and } \quad n \leq \alpha<(m+1) n .
$$

We will estimate $J_{m+1, \alpha}\left(f_{1}, \ldots, f_{m+1}\right)$. We have the following:

$$
\begin{aligned}
& \text { If } \frac{(m+1) n}{m} \leq \alpha<(m+1) n, \quad \text { then } \frac{1}{p_{1}}+\cdots+\frac{1}{p_{m}} \geq 1 . \\
& \text { If } n \leq \alpha<\frac{(m+1) n}{m}, \text { then } \frac{1}{p_{m}}+\frac{1}{p_{m+1}}<1 .
\end{aligned}
$$

The case (13): $(m+1) n / m \leq \alpha<(m+1) n$.

By Hölder's inequality we have

$$
J_{m+1, \alpha}\left(f_{1}, \ldots, f_{m+1}\right) \leq C\left\|f_{m+1}\right\|_{p_{m+1}} J_{m, \alpha-n / p_{m+1}}\left(f_{1}, \ldots, f_{m}\right) .
$$

Since $n \leq \alpha-n / p_{m+1}<m n$ and $1 / p_{1}+\cdots+1 / p_{m}=\left(\alpha-n / p_{m+1}\right) / n$, we obtain the desired result by the hypothesis of induction.

The case (14): $n \leq \alpha<(m+1) n / m$. 
We write

$$
J_{m+1, \alpha}\left(f_{1}, \ldots, f_{m+1}\right)=\int_{\left|y_{m+1}\right| \leq\left|y_{m}\right|}+\int_{\left|y_{m+1}\right| \geq\left|y_{m}\right|}=: J^{1}+J^{2} .
$$

It suffices to estimate $J^{1}$. We have

$$
J^{1} \leq C J_{m, \alpha}\left(f_{1}, \ldots, f_{m-1}, f_{m} \cdot M f_{m+1}\right) .
$$

If $1 / r=1 / p_{m}+1 / p_{m+1}$, then $r>1$. By the hypothesis of induction we obtain

$$
J_{m, \alpha}\left(f_{1}, \ldots, f_{m-1}, f_{m} \cdot M f_{m+1}\right) \leq C \prod_{i=1}^{m-1}\left\|f_{i}\right\|_{p_{i}}\left\|f_{m} \cdot M f_{m+1}\right\|_{r} \leq C \prod_{i=1}^{m+1}\left\|f_{i}\right\|_{p_{i}} .
$$

An outline of the proof of Theorem 1 for $m \geq 4$ and $\alpha=k n$. First we prove the following:

$$
\left\|I_{m,(m-1) n}\left(f_{1}, \ldots, f_{m}\right)\right\|_{q} \leq C \prod_{i=1}^{m-1}\left\|f_{i}\right\|_{1}\left\|f_{m}\right\|_{p_{m}} \quad \text { where } \quad 1<p_{m}<\infty .
$$

Note that $q=p_{m}$, therefore $q>1$. For any nonnegative function $g \in L^{q^{\prime}}$, we estimate

$$
\int I_{m,(m-1) n}\left(f_{1}, \ldots, f_{m}\right)(x) g(x) d x .
$$

The proof is reduced to the $L^{\infty}$ estimate of $I_{m-1,(m-2) n}\left(f_{3}, \ldots, f_{m}, g\right)\left(y_{2}\right)$ (see (10)). Applying Corollary 2 for the indices $\underbrace{1, \ldots, 1}_{m-3}, p_{m}, q^{\prime}$, we obtain the desired result.

In general cases we use induction on $m$. Let $m \geq 3$ be fixed. We assume that for any $1 \leq k \leq m-1$,

$$
\left\|I_{m, k n}\left(f_{1}, \ldots, f_{m}\right)\right\|_{q} \leq C \prod_{i=1}^{k}\left\|f_{i}\right\|_{1} \prod_{i=k+1}^{m}\left\|f_{i}\right\|_{p_{i}}
$$

where $1 / q=\sum_{i=k+1}^{m} 1 / p_{i}$.

We will estimate $I_{m+1, k n}\left(f_{1}, \ldots, f_{m+1}\right)$ where $1 / q=\sum_{i=k+1}^{m+1} 1 / p_{i}$ and $1 \leq k \leq$ $m$. By (15) it suffices to consider the cases when $1 \leq k \leq m-1$. Then $p_{m}, p_{m+1}>1$. We write

$$
\begin{aligned}
I_{m+1, k n}\left(f_{1}, \ldots, f_{m+1}\right)(x) & =\int_{\left|y_{m}\right| \leq\left|y_{m+1}\right|} d y_{1} \cdots d y_{m+1} \\
& +\int_{\left|y_{m}\right| \geq\left|y_{m+1}\right|} d y_{1} \cdots d y_{m+1}=: I^{1}(x)+I^{2}(x) .
\end{aligned}
$$

We have

$$
\begin{aligned}
& I^{1}(x) \leq C M f_{m}(x) \cdot I_{m, k n}\left(f_{1}, \ldots, f_{m-1}, f_{m+1}\right)(x), \\
& I^{2}(x) \leq C M f_{m+1}(x) \cdot I_{m, k n}\left(f_{1}, \ldots, f_{m}\right)(x) .
\end{aligned}
$$

We obtain the desired result by Hölder's inequality and the hypothesis of induction. 


\section{Counterexamples}

We show that the conditions (11) $\sim$ (4) are optimal by giving counterexamples. We consider the case when $n=1$ and $m=3$. Let

$$
I_{3, \alpha}\left(f_{1}, f_{2}, f_{3}\right)(x):=\int_{\left(\mathbb{R}^{1}\right)^{3}} \frac{f_{1}\left(y_{1}\right) f_{2}\left(y_{2}\right) f_{3}\left(y_{3}\right)}{\left(\left|x-y_{1}\right|+\left|x-y_{2}\right|+\left|x-y_{3}\right|\right)^{3-\alpha}} d y_{1} d y_{2} d y_{3},
$$

where $0<\alpha<3$ and $1<p_{1} \leq p_{2} \leq p_{3}<\infty$.

\section{Counterexamples for Theorem 1 and Corollary 1.}

If $0<\alpha<1$ and $1 / q=1+1 / p_{2}+1 / p_{3}-\alpha$, then $I_{3, \alpha}: L^{1} \times L^{p_{2}} \times L^{p_{3}} \nrightarrow L^{q}$.

If $0<\alpha<2$, and $1 / q=2+1 / p_{3}-\alpha$, then

$I_{3, \alpha}: L^{1} \times L^{1} \times L^{p_{3}} \nrightarrow L^{q}$.

If $0<\alpha<1$ and $1 / q=1+1 / p_{2}-\alpha$, then

$I_{3, \alpha}: L^{1} \times L^{p_{2}} \times L^{\infty} \nrightarrow L^{q}$.

If $1 / q=3-\alpha$, then $I_{3, \alpha}: L^{1} \times L^{1} \times L^{1} \nrightarrow L^{q}$.

If $1 / q=2-\alpha$, then $I_{3, \alpha}: L^{1} \times L^{1} \times L^{\infty} \nrightarrow L^{q}$.

If $1 / q=1-\alpha$, then $I_{3, \alpha}: L^{1} \times L^{\infty} \times L^{\infty} \nrightarrow L^{q}$.

Counterexamples for Theorem 2 and Corollary 2.

If $0<\alpha<1$ and $1 / p_{1}+1 / p_{2}+1 / p_{3}=\alpha$, then $I_{3, \alpha}: L^{p_{1}} \times L^{p_{2}} \times L^{p_{3}} \nrightarrow L^{\infty}$.

If $0<\alpha<2$ and $1+1 / p_{2}+1 / p_{3}=\alpha, \quad$ then

$I_{3, \alpha}: L^{1} \times L^{p_{2}} \times L^{p_{3}} \nrightarrow L^{\infty}$.

If $0<\alpha<1$ and $1 / p_{1}+1 / p_{2}=\alpha$, then

$I_{3, \alpha}: L^{p_{1}} \times L^{p_{2}} \times L^{\infty} \nrightarrow L^{\infty}$.

If $1 / p_{1}=\alpha$, then $I_{3, \alpha}: L^{p_{1}} \times L^{\infty} \times L^{\infty} \nrightarrow L^{\infty}$.

If $2+1 / p_{3}=\alpha$, then $I_{3, \alpha}: L^{1} \times L^{1} \times L^{p_{3}} \nrightarrow L^{\infty}$.

If $1+1 / p_{2}=\alpha$, then $I_{3, \alpha}: L^{1} \times L^{p_{2}} \times L^{\infty} \nrightarrow L^{\infty}$.

Proof. (16). Let $\varepsilon=\left(q\left(1 / p_{2}+1 / p_{3}\right)\right)^{-1}$. Since $\alpha<1$ we have $\varepsilon>1$. We define

$$
f_{1}(x)=\chi_{(0,1)}(x) \text { and } f_{i}(x)=x^{-1 / p_{i}}(\log x)^{-\varepsilon / p_{i}} \chi_{\{x \geq 10\}}(x) \text { for } i=2,3,
$$

where $\chi_{E}$ is the characteristic function of a set $E$. Then $f_{1} \in L^{1}, f_{2} \in L^{p_{2}}$ and $f_{3} \in L^{p_{3}}$. If $x \geq 10$, we have

$$
\begin{aligned}
I_{3, \alpha}\left(f_{1}, f_{2}, f_{3}\right)(x) & \geq C \int_{\left(\mathbb{R}^{1}\right)^{2}} \frac{f_{2}\left(y_{2}\right) f_{3}\left(y_{3}\right) d y_{2} d y_{3}}{\left(|x|+\left|x-y_{2}\right|+\left|x-y_{3}\right|\right)^{3-\alpha}} \\
& \geq C \int_{2 x}^{3 x} d y_{2} \int_{2 x}^{3 x} \frac{f_{2}\left(y_{2}\right) f_{3}\left(y_{3}\right)}{\left(|x|+\left|x-y_{2}\right|+\left|x-y_{3}\right|\right)^{3-\alpha}} d y_{3} \\
& \geq C x^{-1 / q}(\log x)^{-1 / q} \notin L^{q} .
\end{aligned}
$$

(17). Let

$$
f_{i}(x)=\chi_{(0,1)}(x) \text { for } i=1,2 \text { and } f_{3}(x)=x^{-1 / p_{3}}(\log x)^{-1 / q} \chi_{\{x \geq 10\}}(x) .
$$


Since $p_{3}>q$ we have $f_{3} \in L^{p_{3}}$. If $x \geq 10$, we have

$$
I_{3, \alpha}\left(f_{1}, f_{2}, f_{3}\right)(x) \geq C \int_{2 x}^{3 x} \frac{f_{3}\left(y_{3}\right) d y_{3}}{\left(|x|+\left|x-y_{3}\right|\right)^{3-\alpha}} \geq C x^{-1 / q}(\log x)^{-1 / q} \notin L^{q} .
$$

(18). Let

$$
f_{1}(x)=\chi_{(0,1)}(x), f_{2}(x)=x^{-1 / p_{2}}(\log x)^{-1 / q} \chi_{\{x \geq 10\}}(x) \text { and } f_{3} \equiv 1 .
$$

(19). Let $f_{i}(x)=\chi_{(0,1)}(x)$ for $i=1,2,3$.

(20). Let $f_{i}(x)=\chi_{(0,1)}(x)$ for $i=1,2$ and $f_{3} \equiv 1$.

(21). Let $f_{1}(x)=\chi_{(0,1)}(x)$ and $f_{i} \equiv 1$ for $i=2,3$.

(22). Let

$$
\begin{gathered}
f_{i}(x)=x^{-1 / p_{i}}(\log x)^{-/ \alpha p_{i}} \chi_{\{x \geq 10\}}(x) \text { for } i=1,2,3, \\
J_{3, \alpha}\left(f_{1}, f_{2}, f_{3}\right) \geq \int_{10}^{\infty} d y_{1} \int_{y_{1}}^{2 y_{1}} d y_{2} \int_{y_{1}}^{2 y_{1}} d y_{3} \geq C \int_{10}^{\infty} \frac{d y_{1}}{y_{1} \log y_{1}}=\infty .
\end{gathered}
$$

For the definition of $J_{3, \alpha}$, see (6).

(23). Note that $1<\alpha<2$. Let $\varepsilon=\left(1 / p_{2}+1 / p_{3}\right)^{-1}$. Then $\varepsilon>1$. Let

$$
\begin{gathered}
f_{1}(x)=\chi_{(0,1)}(x) \text { and } f_{i}(x)=x^{-1 / p_{i}}(\log x)^{-\varepsilon / p_{i}} \chi_{\{x \geq 10\}}(x) \text { for } i=2,3, \\
J_{3, \alpha}\left(f_{1}, f_{2}, f_{3}\right) \geq C \int_{10}^{\infty} \frac{1}{y_{3} \log y_{3}} d y_{3}=\infty .
\end{gathered}
$$

(24). Let

$$
f_{i}(x)=x^{-1 / p_{i}}(\log x)^{-/ \alpha p_{i}} \chi_{\{x \geq 10\}}(x) \text { for } i=1,2 \text { and } f_{3} \equiv 1 .
$$

(25). Let

$$
f_{1}(x)=x^{-1 / p_{1}}(\log x)^{-1} \chi_{\{x \geq 10\}} \text { and } f_{i} \equiv 1 \text { for } i=2,3 .
$$

(26). Let

$$
\begin{gathered}
f_{i}(x)=\chi_{(0,1)}(x) \text { for } i=1,2 \text { and } f_{3}(x)=x^{-1 / p_{3}}(\log x)^{-1} \chi_{\{x \geq 10\}}(x), \\
J_{3, \alpha}\left(f_{1}, f_{2}, f_{3}\right) \geq C \int_{10}^{\infty} \frac{f_{3}\left(y_{3}\right) d y_{3}}{y_{3}^{3-\alpha}}=\infty .
\end{gathered}
$$

(27). Note that $\alpha<2$. Let

$$
\begin{gathered}
f_{1}(x)=\chi_{(0,1)}(x), f_{2}(x)=x^{-1 / p_{2}}(\log x)^{-1} \chi_{\{x \geq 10\}}(x) \text { and } f_{3} \equiv 1, \\
J_{3, \alpha}\left(f_{1}, f_{2}, f_{3}\right) \geq C \int_{10}^{\infty} \frac{f_{2}\left(y_{2}\right) d y_{2}}{y_{2}^{2-\alpha}}=\infty .
\end{gathered}
$$




\section{REFERENCES}

[1] Loukas Grafakos, Modern Fourier analysis, 2nd ed., Graduate Texts in Mathematics, vol. 250, Springer, New York, 2009. MR2463316

[2] Loukas Grafakos and Rodolfo H. Torres, Multilinear Calderón-Zygmund theory, Adv. Math. 165 (2002), no. 1, 124-164, DOI 10.1006/aima.2001.2028. MR.1880324

[3] G. H. Hardy, J. E. Littlewood, and G. Pólya, Inequalities, Cambridge, at the University Press, 1952. 2d ed. MR.0046395

[4] Carlos E. Kenig and Elias M. Stein, Multilinear estimates and fractional integration, Math. Res. Lett. 6 (1999), no. 1, 1-15, DOI 10.4310/MRL.1999.v6.n1.a1. MR1682725

[5] Michael Lacey and Christoph Thiele, $L^{p}$ estimates on the bilinear Hilbert transform for $2<$ $p<\infty$, Ann. of Math. (2) 146 (1997), no. 3, 693-724, DOI 10.2307/2952458. MR 1491450

[6] Lin Tang, Endpoint estimates for multilinear fractional integrals, J. Aust. Math. Soc. 84 (2008), no. 3, 419-429, DOI 10.1017/S1446788708000724. MR2453689

Department of Mathematics, School of Science, Tokai University, Hiratsuka, KanaGAWA 259-1299 JAPAN

E-mail address: komori@tokai-u.jp 